Original Research

\title{
Comparison of Strain Index (SI) and ACGIH-HAL in Assessing the Risk of Upper Extremities Disorders and Prediction of Carpal Tunnel Syndrome in Butchers
}

\author{
Fakhradin Ghasemi $^{1}$, Kamran GholamiZadeh $^{2^{*}}$, Amin Doosti-Irani ${ }^{3}$, Ramin Rahmani $^{2}$
}

1. Assistant Professor, Department of Ergonomics, Occupational Health and Safety Research Center, School of Public Health, Hamadan University of Medical Sciences, Hamadan, Iran

2. MSc Student, Department of Occupational Health Engineering, Student Research Committee, School of Public Health, Hamadan University of Medical Sciences, Hamadan, Iran

3. Assistant Professor, Department of Epidemiology, Health Sciences Research Center, School of Public Health, Hamadan University of Medical Sciences, Hamadan, Iran

\begin{tabular}{ll}
\hline \multicolumn{2}{c}{ Article Info } \\
\hline Original Article \\
Received: & $2019 / 02 / 05$ \\
Accepted: & $2019 / 03 / 12$ \\
Published Online: & $2019 / 03 / 12$
\end{tabular}

DOI: $10.30699 /$ jergon.6.4.1

Use your device to scan and read the article online

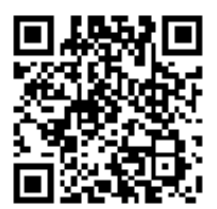

Corresponding Information

Kamran GholamiZadeh, MSc Student, Department of Occupational Health Engineering, Student Research Committee, School of Public Health, Hamadan University of Medical Sciences, Hamadan, Iran

Email:

kamrangholamizade1373@gmail.com

\section{Abstract}

Background and Objectives: Upper extremities are very vulnerable to work-related musculoskeletal disorders and selecting the best technique for assessing their exposure to ergonomic risk factors is of pivotal importance. This study aimed to compare two techniques of SI and ACGIH-HAL and assess their relationship with carpal tunnel syndrome (CTS) among butchers.

Methods: The study population was all butchers in Hamadan, Iran. After observing the activities, the assessments were conducted using both techniques. The severity of CTS syndromes was assessed using Boston questionnaire. The agreement between the techniques was investigated using Kappa coefficient. The association between the risk levels obtained from the techniques with the severity of CTS syndromes were assessed by regression analysis and fixing the effect of personal factors such as age, body mass index, and wrist ratio index.

Results: A total number of 152 butchers were evaluated. SI and ACGIH-HAL techniques respectively recognized 76 and 102 cases as low risk (agreement in 69 cases), 40 and 27 cases as moderate risk (agreement in 8 cases), and 36 and 23 cases as high risk (agreement in 18 cases). The Kappa coefficient between two techniques was $0.36(P<0.001)$. By fixing the effects of age, body mass index, and wrist ratio index, both techniques had significant association with the severity of CTS syndromes. However, the association between SI and severity of CTS syndrome was higher than that of ACGIH-HAL.

Conclusion: SI had a higher estimate of risk than ACGIH-HAL. It seems that the predictive ability of SI for the severity of CTS syndrome is better than that of ACGIH-HAL.

Keywords: Risk assessment, Upper extremities, Carpal Tunnel Syndrome

Copyright $@$ C 2019, Journal of Ergonomics. This is an open-access article distributed under the terms of the Creative Commons Attribution-noncommercial 4.0 International License which permits copy and redistribute the material just in noncommercial usages, provided the original work is properly cited

How to Cite This Article:

Ghasemi F, Gholamizadeh K, Dousti-Irani A, Ramin R. Comparison of Strain Index (SI) and ACGIH-HAL in Assessing the Risk of Upper Extremities Disorders and Prediction of Carpal Tunnel Syndrome in Butchers. Iran J Ergon. 2019; 6 (4) :1-8 
مقالة يزوهشى

مقايسة دو تكنيك SI و ACGIH-HAL در ارزيابى اختلالات اندامهاى فوقانى انتهايى و ييشبينى سندرم تونل كاري در قصابان

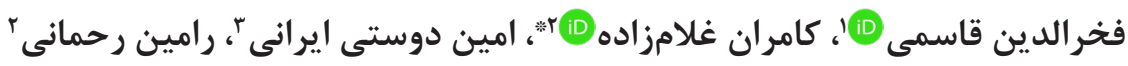

ا. استاديار، كروه اركونومى، مركز تحقيقات ايمنى و بهداشت شغلى، دانشكدة بهداشت، دانشعاه علوم يزشكى همدان، همدان، ايران

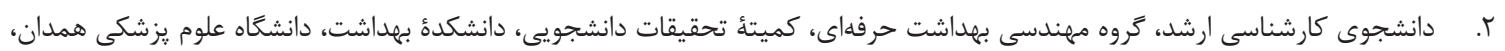

همدان، ايران

". استاديار، تروه إِيدميولوزى، مركز تحقيقات علوم بهداشتى، دانشكدة بهداشت، دانشكاه علوم يزشكى همدان، همدان، ايران

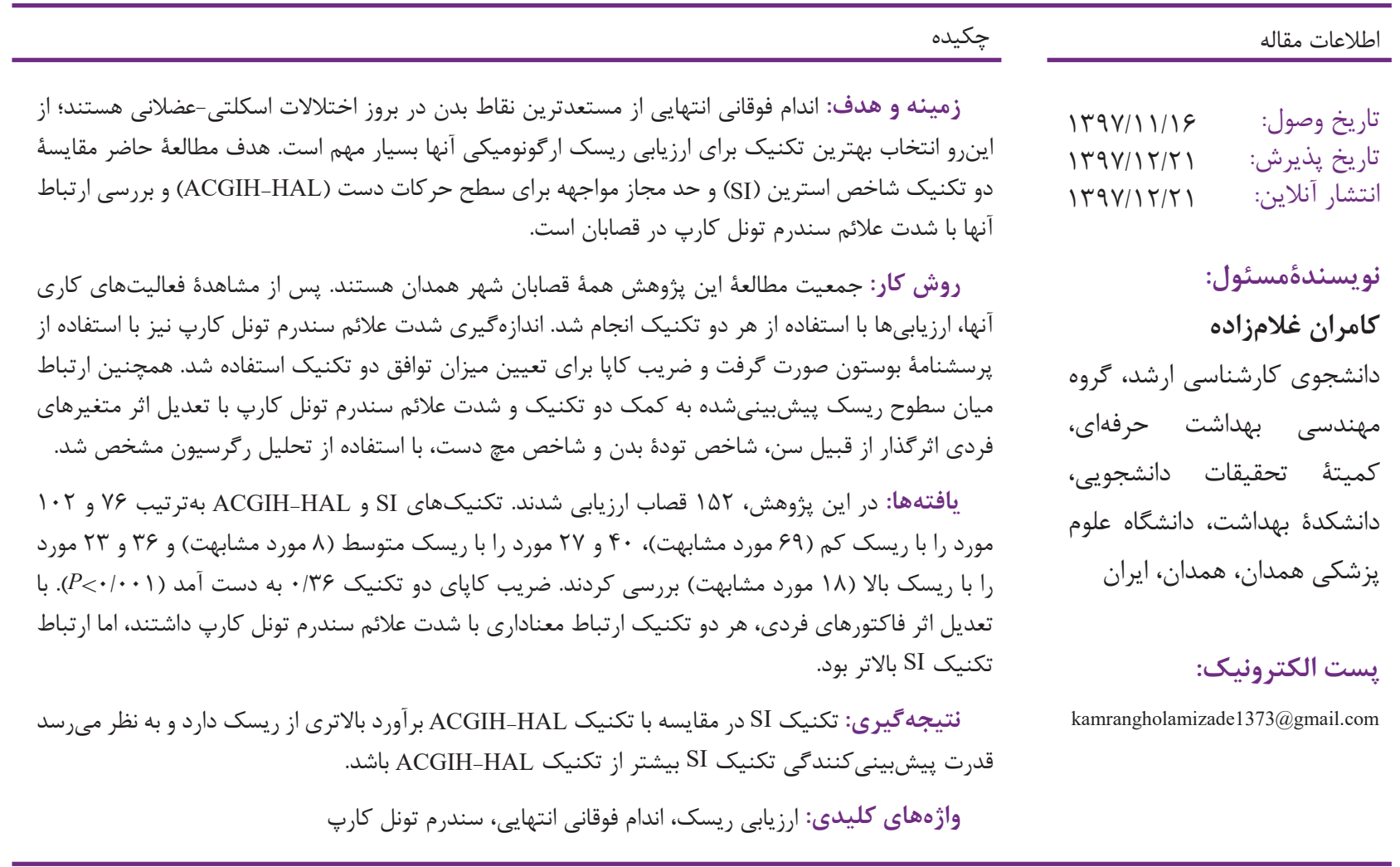

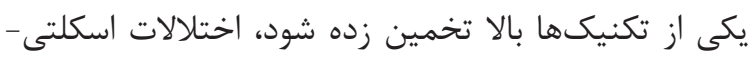

مقلمهـ

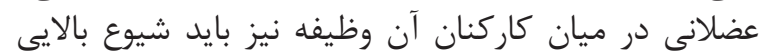

داشته باشد [ب].

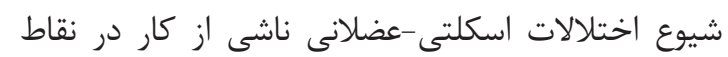

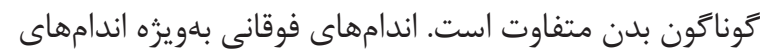

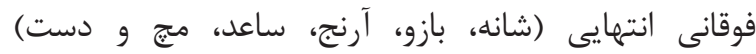

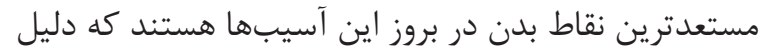

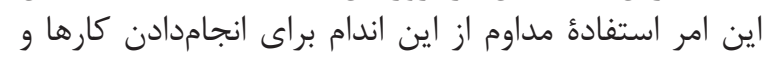

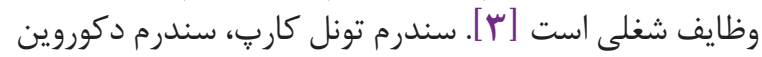

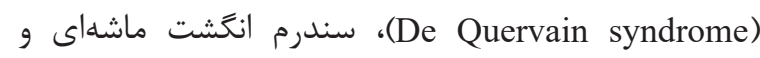

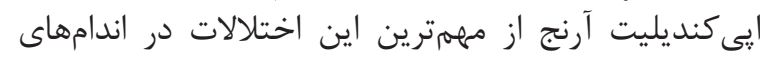

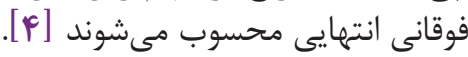

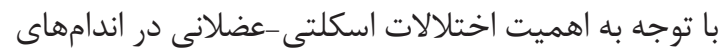

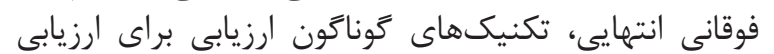

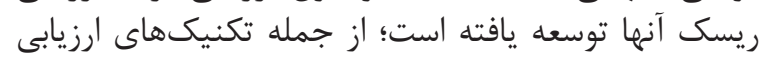

اختلالات اسكلتى-عضلانى ناشى از كار يكى از مهامهترين

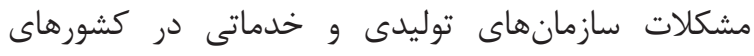

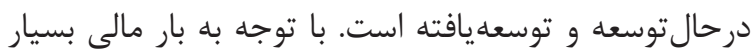

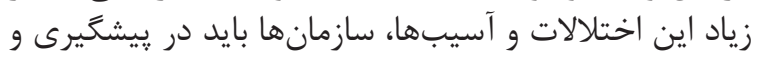

مديريت آنها كوشا باشند [1]]

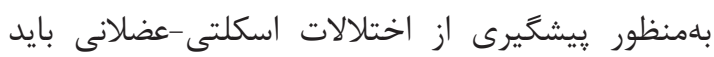

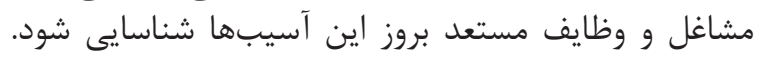

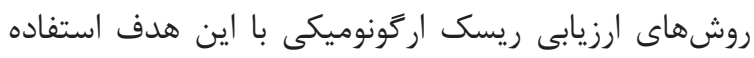

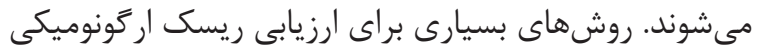

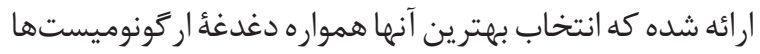

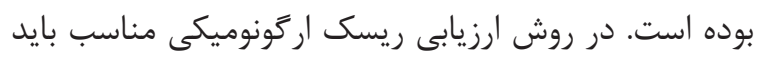

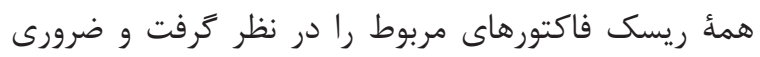

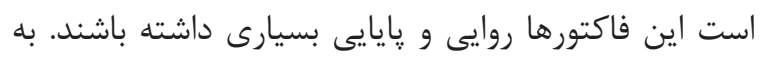

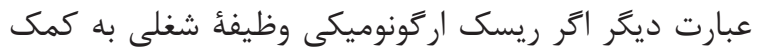




\section{$\mathrm{SI}=\mathrm{IE} \times \mathrm{DE} \times \mathrm{EM} \times \mathrm{HWP} \times \mathrm{SW} \times \mathrm{DD}$}

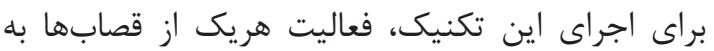

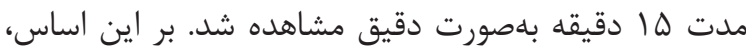

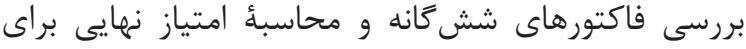

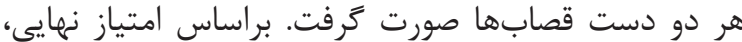

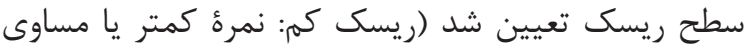

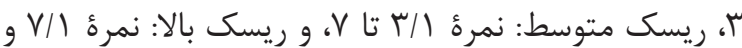

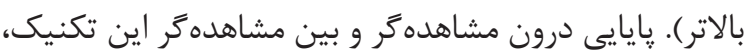

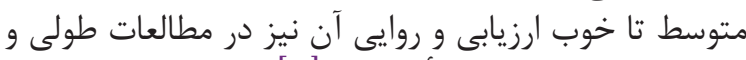
هم در مطالعات مقطعى تأييد شد آن آن.

\section{ACGIH-HAL شاخص}

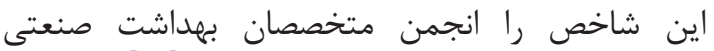

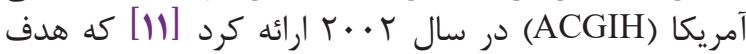

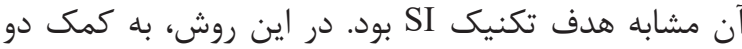

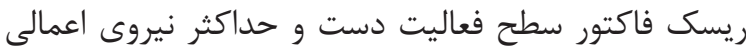

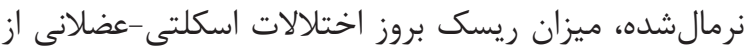

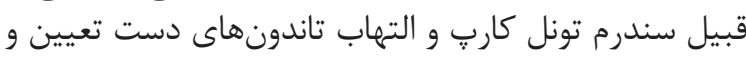

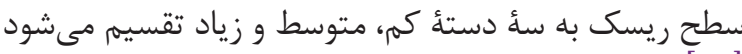

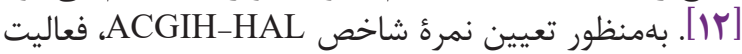

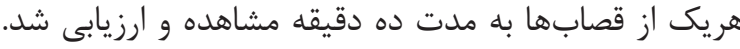

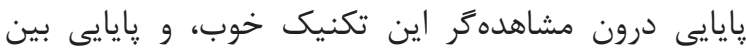

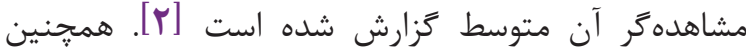

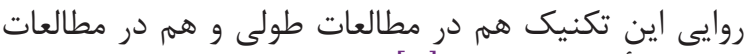

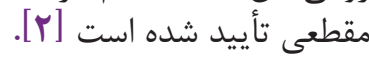

\section{شدت علائم سندرم تونل كارٍ}

در اين مطالعه، از يرسشنامة سندرم تونل كارِّ با عنوان

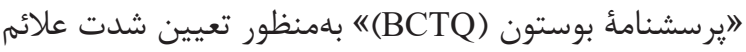

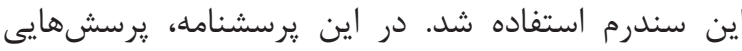

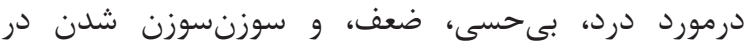

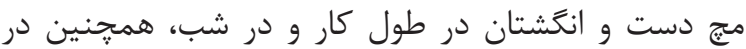

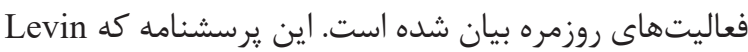

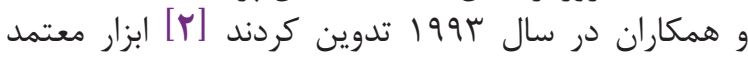

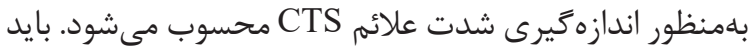

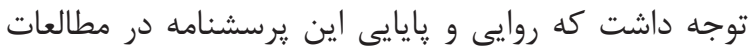

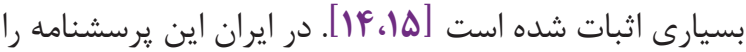

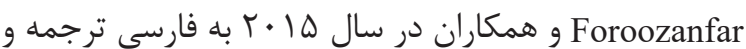

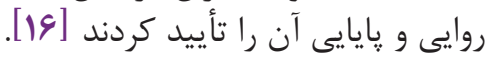

$$
\text { آناليز آمارى }
$$

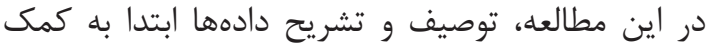

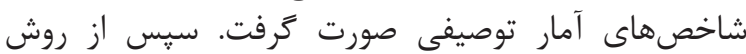

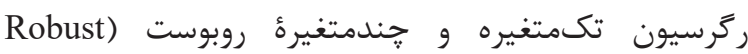

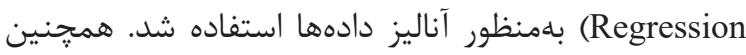

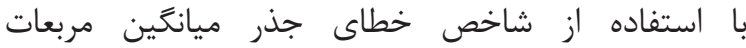
مقايسٔ خطاهاى مeot Mean Square Error, RMSE) مدلهاى رَرسيونى مختلف با يكديخر صورت كرفت.

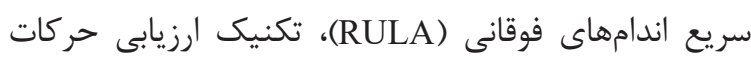

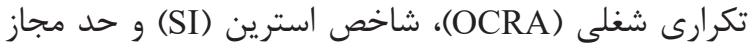

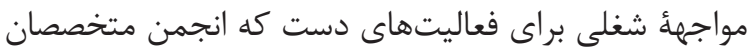

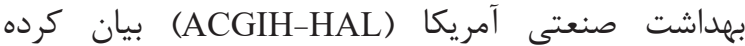
است [F]. در اين ميان، دو شاخص SI و و

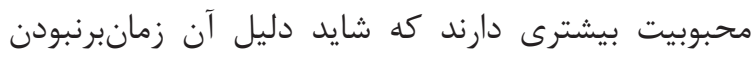

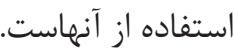

همان كَونه كه بِيش از اين اشاره شد، سندرم تونل كارٍ

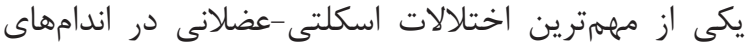

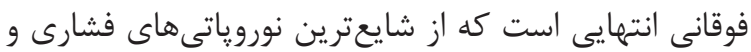

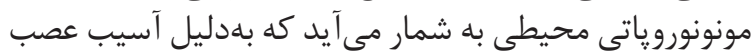

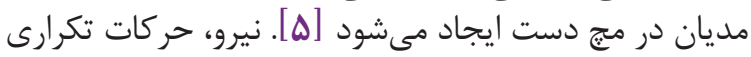

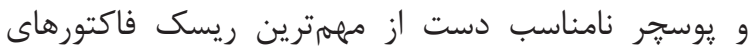

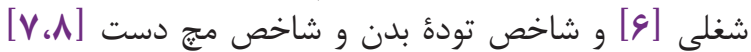

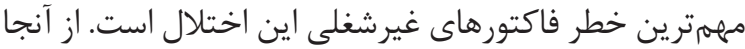

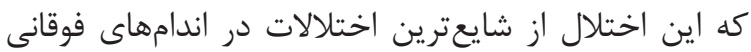

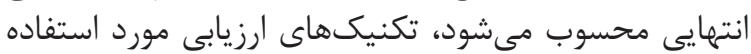

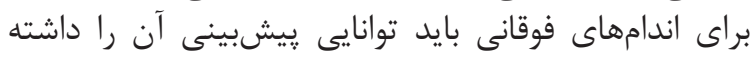

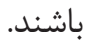

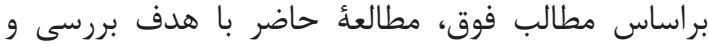

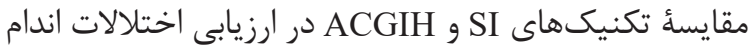

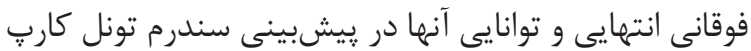

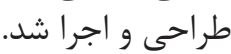

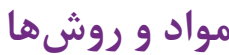

\section{مشاركت كنند}

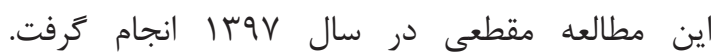

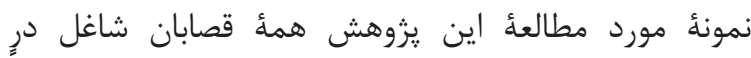

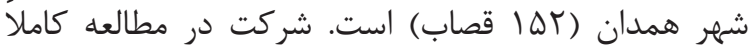

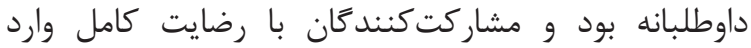

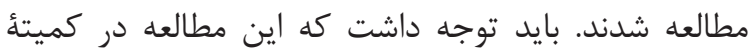

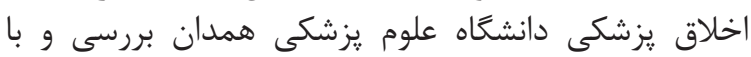

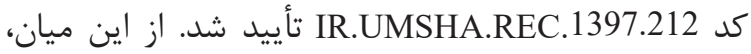

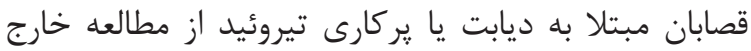

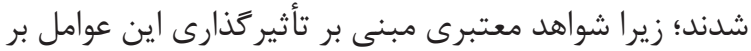

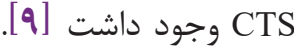

\section{SI تكنيك}

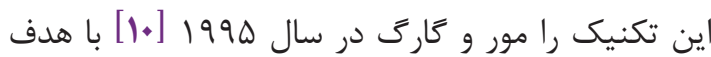

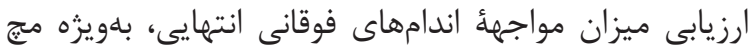

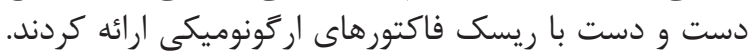

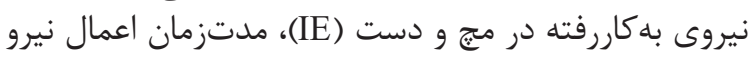

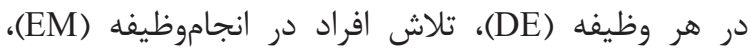

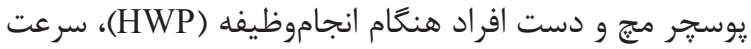

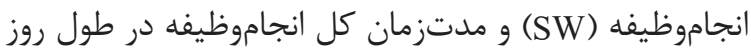

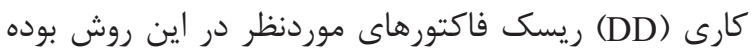

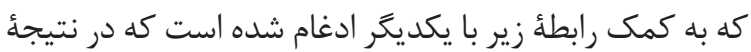

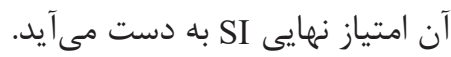


آمده است. بر اين اساس، تكنيكهاى SI و

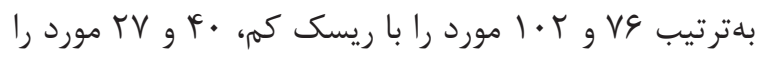

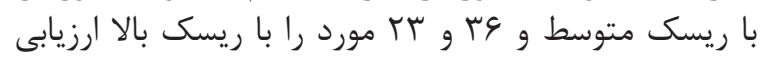
كردند. بdطوركلى، نتايج SI برآورد بيشترى از ريسك دارد.

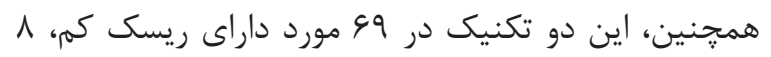

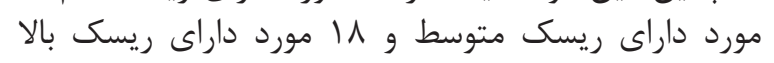

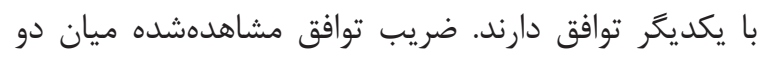

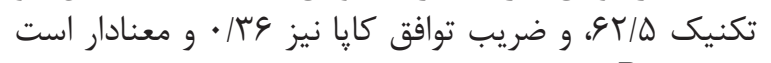

بافته ها $(P<\cdot / \cdot 1)$

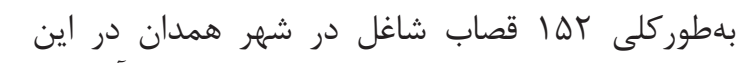

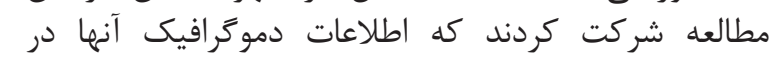

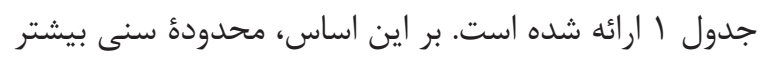

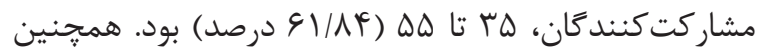

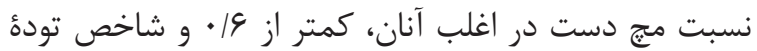

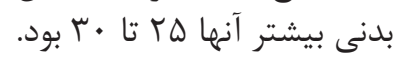

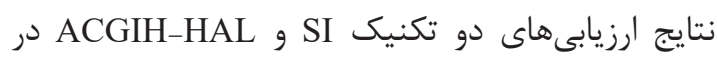
شكل l و ميزان توافق مشاهدهشده دو تكنيك در در جدول

جدول ا. اطلاعات دموكر افيك شركت كنندكان در مطالعه

\begin{tabular}{|c|c|c|}
\hline تعداد (درصد) & طبقهبندى & متغير \\
\hline$(\Delta / r \varepsilon) \wedge$ & $r \Delta \geq$ & \multirow{5}{*}{ سن } \\
\hline rr (TI/V) & $r \Delta-r \Delta$ & \\
\hline$(g / / A F) q F$ & $\Delta \Delta-r \Delta$ & \\
\hline$(11 / 1 \wedge) I V$ & $\Delta \Delta \leq$ & \\
\hline$(1 \cdot \cdot) \mid \Delta T$ & كلى & \\
\hline$(F Y / V \varphi) \& \Delta$ & $\cdot 19 \geq$ & \multirow{4}{*}{ نسبت مج دست } \\
\hline$(r F / T I) \Delta T$ & $\cdot 19_{-} \cdot / V$ & \\
\hline D & $\cdot / V \leq$ & \\
\hline$(1 \cdot \cdot) \mid \Delta r$ & كلى & \\
\hline$\left(\Gamma / \mathcal{T}^{\mu}\right) \mathcal{F}$ & $\mid N / \Delta \geq$ & \multirow{6}{*}{ شاخص تودهُ بدنى } \\
\hline$(\mid r / / \Delta) r \cdot$ & $r r-1 N / \Delta$ & \\
\hline$(19 / V \mu) \Gamma$. & rr-rd & \\
\hline$(\Delta \cdot \mid q T) V V$ & $r \Delta-r$. & \\
\hline$(\mid F / F V) \mid F$ & $r \cdot \leq$ & \\
\hline$(1 \cdots) \mid \Delta r$ & كلى & \\
\hline
\end{tabular}

جدول r. ميزان توافق بهدست آمده از تكنيكهاى SI و ACGIH-HAL

\begin{tabular}{|c|c|c|c|c|}
\hline \multirow{2}{*}{ 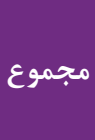 } & \multicolumn{3}{|c|}{ تكنيك ACGIH-HAL } & \multirow{2}{*}{ SI تكنيك } \\
\hline & ريسك بالا & ريسك متوسط & ريسك كم & \\
\hline ve & r & f & 99 & ريسك كم \\
\hline f. & r & $\wedge$ & r. & ريسك متوسط \\
\hline re & 11 & 10 & r & ريسك بالا \\
\hline IDT & r & tr & $1 \cdot r$ & مجموع \\
\hline
\end{tabular}




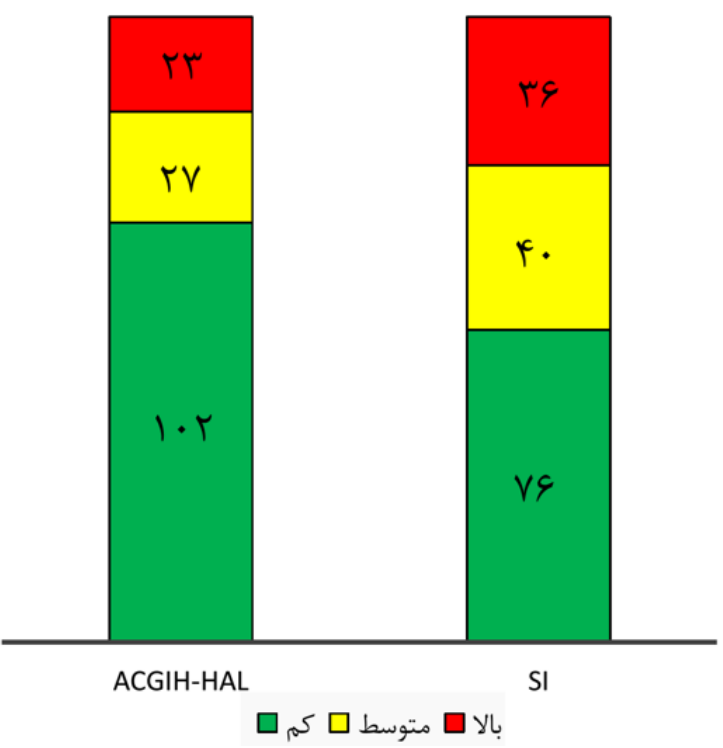

شكل ا. فراوانى سطوح ريسك پِيشبينىشده براى موارد كوناكون به كمك دو تكنيك ACGIH-HAL

بر اين اساس، امتياز SI يِيشبين قوىترى از سن، شاخص

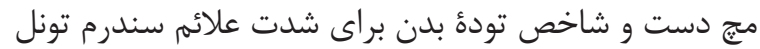
كارب است. - ماست

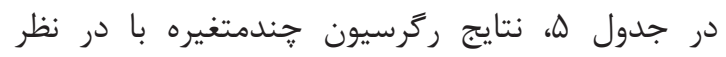

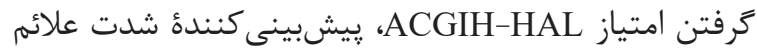

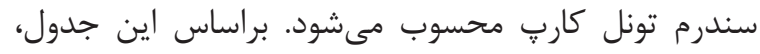

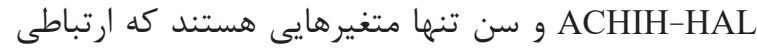
معنادارى با شدت علائم سندرم تونل كارب دارند
نتايج ركرسيون تكمتغيره در جدول بـ آمده است.

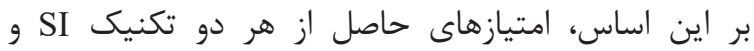
ACGIH-HAL

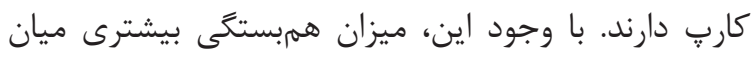

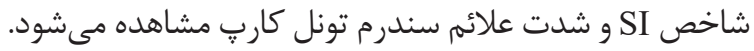

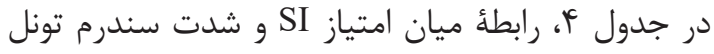

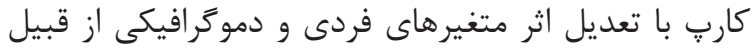
سن، شاخص مج دست و شاخص توده بدن مشاهده مى شودي.

جدول rا. بررسى ارتباط شدت علائم سندرم تونل كارب و سطوح ريسك بهدست آمده از تكنيكهاى SI و ACGIH

\begin{tabular}{|c|c|c|c|c|}
\hline \multicolumn{2}{|c|}{ فاصلة اطمينان (ه د درصد) } & $\boldsymbol{P}$ & ضريب & متغير \\
\hline V/lIT & $\Delta / r \varepsilon V$ & $<\cdot / \cdot \cdot 1$ & $9 / 19$. & SI \\
\hline $9 / 099$ & ए/सq & $<\cdot 1 \cdot .1$ & $\Delta / \cdot M^{\prime}$ & ACGIH-HAL \\
\hline
\end{tabular}

جدول f. بررسى رابطةُ شدت علائم سندرم تونل كارٍ و سطح ريسك بهدست آمده از تكنيك SI با توجه به فاكتورهاى فردى

\begin{tabular}{|c|c|c|c|c|c|}
\hline \multirow[t]{2}{*}{ RMSE } & \multicolumn{2}{|c|}{ فاصلة اطمينان (ه د درصد) } & $P$ & ضريب ل & متغير \\
\hline & $\mathrm{V} / \cdot \wedge \mathrm{F}$ & $\Delta /|f|$ & $<\cdot 1 \cdot \cdot 1$ & $9 / 114$ & SI \\
\hline \multirow{4}{*}{$9 / 49$} &.$- / . K t$ & - & $\cdot \pi v$. &.$\cdot$ HAF & سن \\
\hline & $-9 / 4)$. & १/१Q४ & $\cdot / 94 \lambda$ & . & شاخص مجج دست \\
\hline & $-\cdot / 1 \Lambda$. & $\cdot / 109$ & $\cdot / 9 \cdot 4$ &.$- / .1$. & شاخص تودةٔ بدن \\
\hline & 19/991 & D/99) & $\cdot / \cdot \cdot 1$ & Ir/Ar. & ثابت \\
\hline
\end{tabular}


جدول ه. بر رسى رابطهُ شدت علائم سندرم تونل كارٍ و سطح ريسك بهدست آمده از تكنيك ACGIH-HAL با توجه به فاكتورهاى فردى

\begin{tabular}{|c|c|c|c|c|c|}
\hline RMSE & \multicolumn{2}{|c|}{ فاصلة اطمينان (ه د درصد) } & $P$ & ضريب & متغير \\
\hline \multirow{5}{*}{$\mathrm{V} / \cdot 9$} & Q/qUI & T/QTV & $<\cdot \mid \cdot \cdot 1$ & F/ATq & ACGIH-HAL \\
\hline & . TFr & מr & $\cdot / \cdot 11$ & سז/1. & سن \\
\hline & $\mid \varepsilon / 4 \cdot \Delta$ & $-11 / 999$ & . /Vrq & $r / T r I$ & شاخص مج دست \\
\hline & • T/N & .1111 & $\cdot / T V A$ & -.1148 & شاخص تودهٔ بدن \\
\hline & $r \Delta / r \Lambda \Lambda$ & $p / 9 \wedge q$ & $\cdot / \cdot r$ & $10 / 119$ & ثابت \\
\hline
\end{tabular}

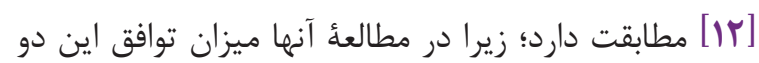

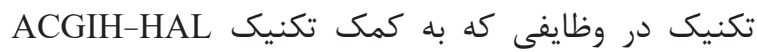

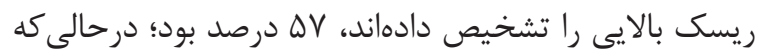

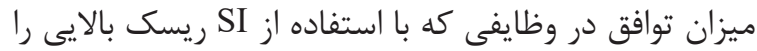

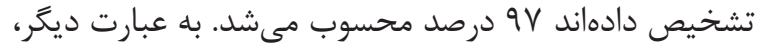

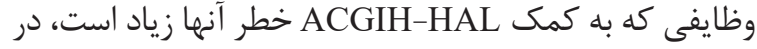

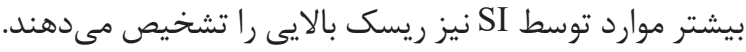

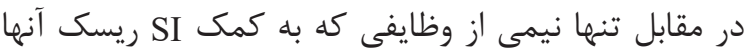
بالاست، توسط ACGIH-HAL نيز نتايج مشابهى را را نشان

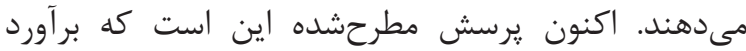

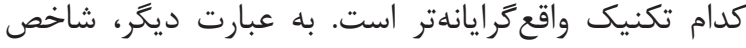

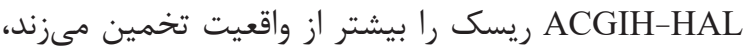

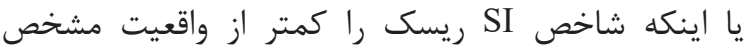

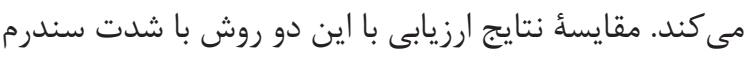

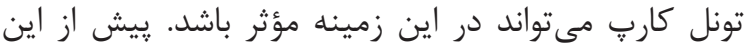

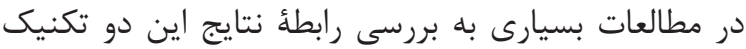

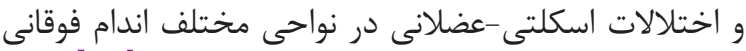

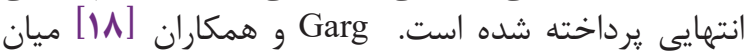

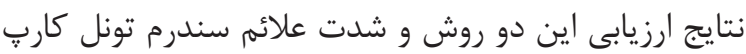

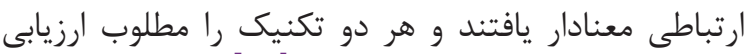

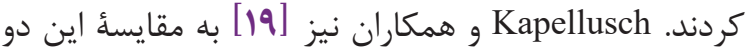

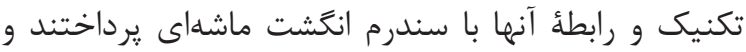

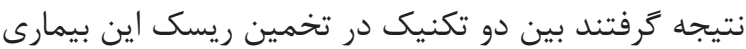

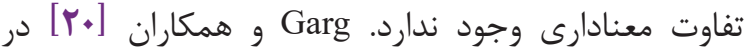

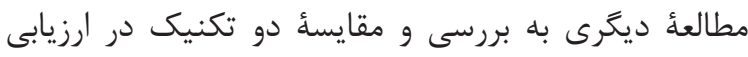

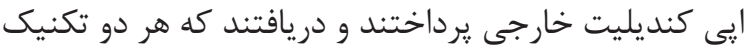

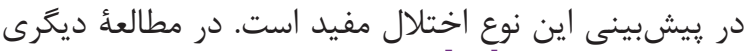
و و Jones Jكاي OCRA RULA، REBA، ACGIH-HAL، SI تكرارى در صنعت جوب را تحليل كردند و نتيجه كرفتند كهال

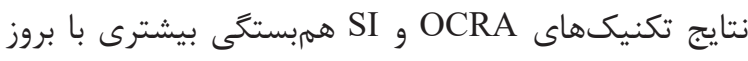

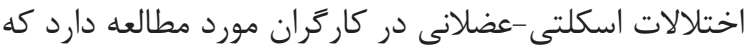

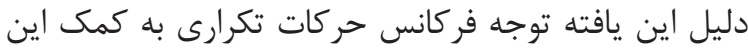
دو تكنيك است.

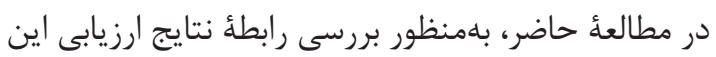

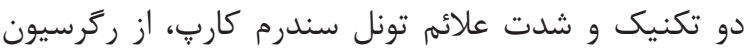

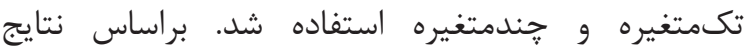

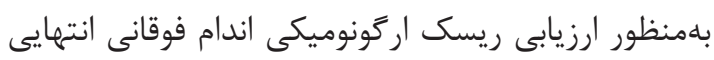

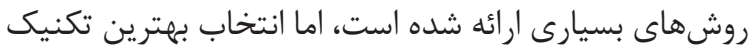

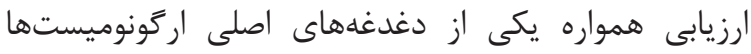

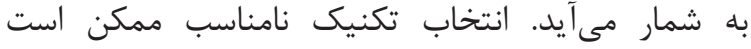

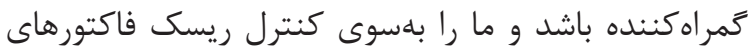

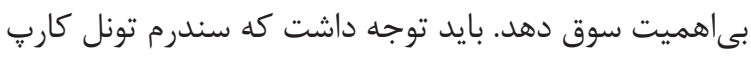

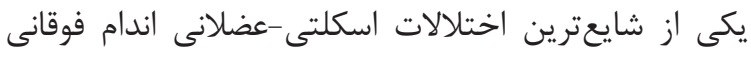

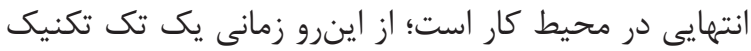

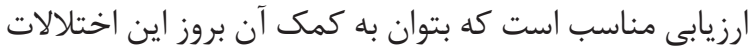

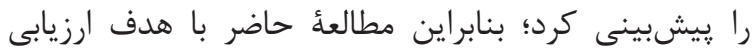
و مقايسٔ دو تكنيك SI و

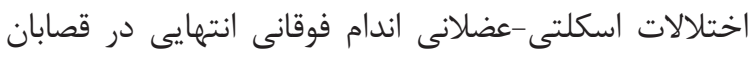

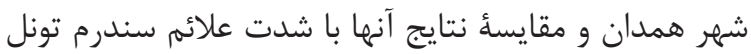
كارب صورت كرفته است.

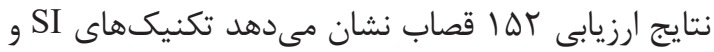

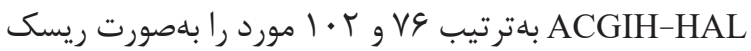

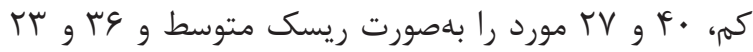

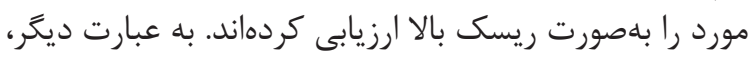

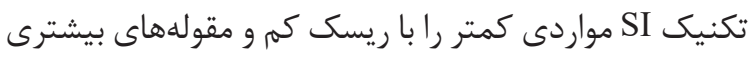

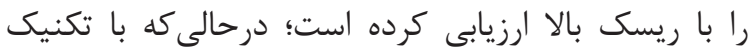
ACGIH-HAL

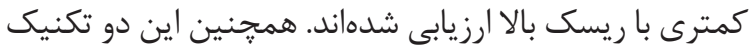

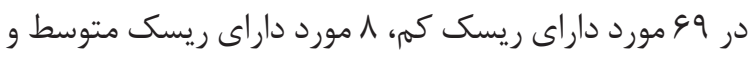

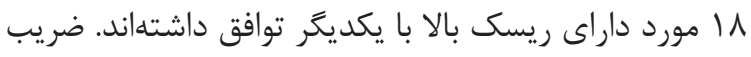

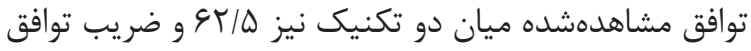

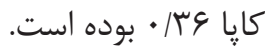

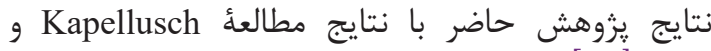

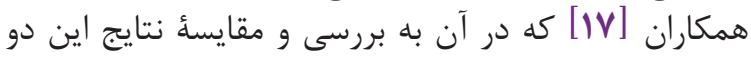

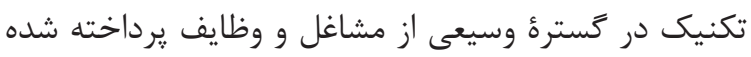

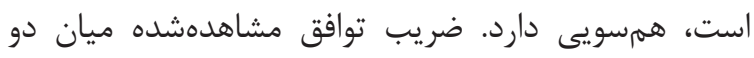

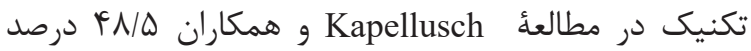

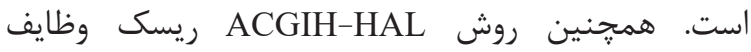

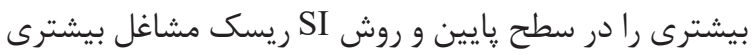

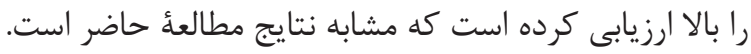

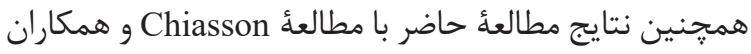




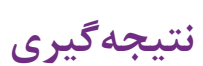

تكنيك SI در مقايسه با تكنيك ACGIH-HAL بر آورد

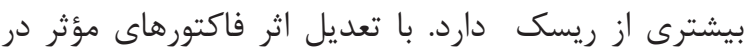

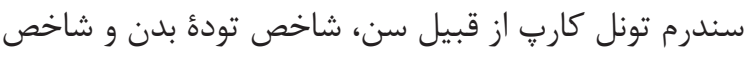

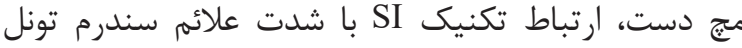
كار乡 از تكنيك دCGIH-HAL بيشتر است.

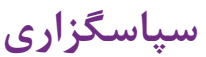

نويسندكان از معاونت تحقيقات و فناورى دانشعاه علوم

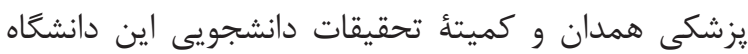

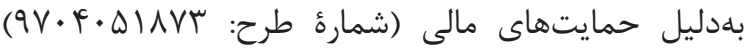
تشكر مى كنند.

$$
\text { تعارض منافع }
$$

بين نويسند

\section{References}

1. Bevan S. Economic impact of musculoskeletal disorders (MSDs) on work in Europe. Best Pract Res Clin Rheumatol. 2015;29(3):356-73. https://doi.org/10.1016/j.berh.2015.08.002 PMID:26612235

2. Takala E-P, Pehkonen I, Forsman M, Hansson G-Å, Mathiassen SE, Neumann WP, et al. Systematic evaluation of observational methods assessing biomechanical exposures at work. Scand J Work Environ Heal. 2010;36(1):3-24. https:// doi.org/10.5271/sjweh.2876

3. Work related musculoskeletal disorders in Great Britain (WRMSDs), 2018, Annual Statistics, Health and Safety Executive.

4. Buckle PW, Devereux Jason J. The nature of work-related neck and upper limbmusculoskeletal disorders. Appl Ergon. 2002;33:207-17. https://doi.org/10.1016/S0003-6870(02)00014-5

5. Cooke ME, Duncan SF. History of carpal tunnel syndrome. InCarpal tunnel syndrome and related median neuropathies 2017 (pp. 7-11). Springer, Cham. https://doi.org/10.1007/978-3-319-570105 2

6. Silverstein BA, Fine LJ, Armstrong TJ. Occupational factors and carpal tunnel syndrome. Am J Ind Med. 1987;11(3):343-58. https://doi. org/10.1002/ajim.4700110310 PMID:3578290

7. Ozcakir S, Sigirli D, Avsaroglu H. High wrist ratio is a risk factor for carpal tunnel syndrome.

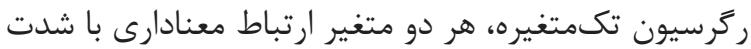

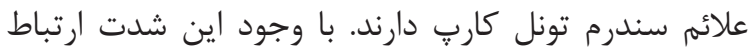
شاخص SI و شدت سندرم تونل كارب بيشتر است. همجنين

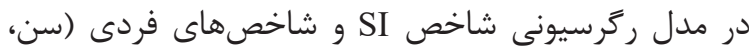

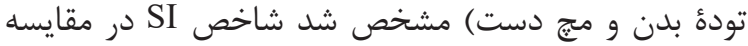

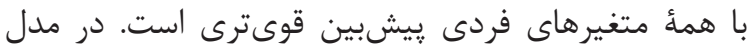

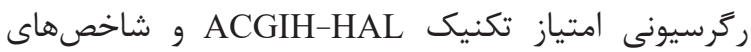
فردى، علاوه بر امتياز اين تكنيك، سن نيز اثنيك اثر معنادارى

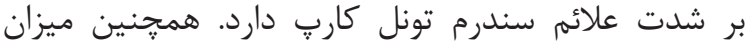
RMSE ACGIH-HAL است. اين نتايج مشابه نتايج مطالعئ كذشته دئه

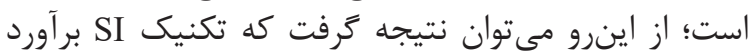

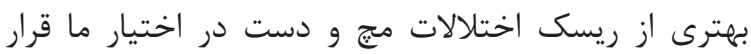

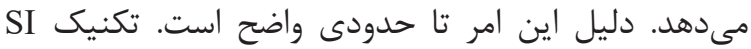

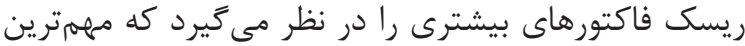

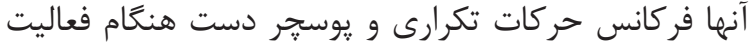
محسوب مىشود كه در وظايف شغلى قصابان برجسته است.

Clin Anat. 2018;31(5):698-701. https://doi. org/10.1002/ca.23198 PMID:29722064

8. Shiri R, Pourmemari MH, Falah-Hassani K, Viikari-Juntura E. The effect of excess body mass on the risk of carpal tunnel syndrome: A meta-analysis of 58 studies. Obes Rev. 2015;16(12):1094-104. https://doi.org/10.1111/ obr.12324 PMID:26395787

9. Padua L, Coraci D, Erra C, Pazzaglia C, Paolasso I, Loreti C, et al. Carpal tunnel syndrome: clinical features, diagnosis, and management. Lancet Neurol [Internet]. 2016;15(12):1273-84. http:// dx.doi.org/10.1016/S1474-4422(16)30231-9

10. Moore JS, Garg A. The Strain Index: A Proposed Method to Analyze Jobs For Risk of Distal Upper Extremity Disorders. Am Ind Hyg Assoc J. 1995;56(5):443-58. https:// doi.org/10.1080/15428119591016863 PMID:7754975

11. American Conference of Governmental Industrial Hygienists (ACGIH), 2002. Hand Activity Level (HAL). Threshold Limit Values for Chemical Substances and Physical Agents \& Biological Exposure Indices. ACGIH, Cincinnati, pp. $112 \mathrm{e} 114$.

12. Chiasson M ève, Imbeau D, Aubry K, Delisle A. Comparing the results of eight methods used to evaluate risk factors associated with musculoskeletal disorders. Int J Ind Ergon. 2012;42(5):478- 
88. https://doi.org/10.1016/j.ergon.2012.07.003

13. Levine D, Simmons B, Koris M, Daltroy L, Hohl G, Fossel A, et al. A self-administered questionnaire for the assessment of severity of symptoms and functional status in carpal tunnel syndrome. J Bone Jt Surg. 1993;75(11):1585-92. https:// doi.org/10.2106/00004623-199311000-00002 PMID: 8245050

14. Lue YJ, Lu YM, Lin GT, Liu YF. Validation of the Chinese version of the boston carpal tunnel questionnaire. J Occup Rehabil. 2014;24(1):13945. https://doi.org/10.1007/s10926-013-9438-9 PMID: 23546645

15. Mondelli M, Reale F, Sicurelli F, Padua L, Senese AO. Relationship between the self-administered Boston questionnaire and electrophysiological findings in follow-up of surgically-treated carpal tunnel syndrome. J Hand Surg Am. 2000;25(2):128-34. https://doi.org/10.1054/ jhsb.2000.0361 PMID: 11062567

16. Foroozanfar Z, Ebrahimi H, Khanjani N. Validity and Reliability of the Persian Boston Questionnaire in Diabetic Patients with Carpal Tunnel Syndrome. J Neyshabur Univ Med Sci. 2015;2(5):50-6.

17. Kapellusch JM, Bao SS, Silverstein BA, Merryweather AS, Thiese MS, Hegmann KT, et al. Risk assessments using the strain index and the TLV for HAL, part I: Task and multi-task job exposure classifications. J Occup Environ Hyg. 2017;14(12):1011-9. https://doi.org/10.1080/15 459624.2017.1366037 PMID: $\underline{28825893}$

18. Garg A, Kapellusch J, Hegmann K, Wertsch J, Merryweather A, Deckow-Schaefer G, et al. The Strain Index (SI) and Threshold Limit Value (TLV) for Hand Activity Level (HAL): Risk of carpal tunnel syndrome (CTS) in a prospective cohort. Ergonomics. 2012;55(4):396-414. https://doi.org/10.1080/00140139.2011.644328 PMID:22397385

19. Kapellusch JM, Garg A, Hegmann KT, Thiese MS, Malloy EJ. The strain index and ACGIH TLV for HAL: Risk of trigger digit in the WISTAH prospective cohort. Hum Factors. 2014;56(1):98111. https://doi.org/10.1177/0018720813493115
PMID:24669546

20. Garg A, Kapellusch JM, Hegmann KT, Thiese MS, Merryweather AS, Wang YC, et al. The Strain Index and TLV for HAL: Risk of lateral epicondylitis in a prospective cohort. Am J Ind Med. 2014;57(3):286-302. https://doi. org/10.1002/ajim.22279

21. Jones T, Kumar S. Comparison of ergonomic risk assessments in a repetitive high-risk sawmill occupation: Saw-filer. Int J Ind Ergon. 2007;37(9-10):744-53. https://doi.org/10.1016/j. ergon.2007.05.005 